\title{
ESTIMULAÇÃO CEREBRAL CONTÍNUA (DBS) TALÂMICA PARA CONTROLE DO TREMOR
}

\author{
José Augusto Nasser1,2, Asdrubal Falavigna', Armando Alaminos', \\ Antônio de Pádua Bonatellỉ, Fernando Ferraz ${ }^{3}$
}

\begin{abstract}
RESUMO - Objetivo: Apresentamos resultados da estimulação contínua do núcleo ventral intermédio (VIM) talâmico para o controle do tremor. Método: Quatro pacientes foram selecionados no período de outubro de 1999 a janeiro de 2001 com tremor incapacitante refratário à farmacoterapia. Dois pacientes apresentavam tremor essencial (TE) bilateral e 2 pacientes tremor de repouso por doença de Parkinson (DP), um à direita e outro à esquerda. Após avaliação sistemática, foram submetidos ao implante de eletrodo talâmico, modelo DBS 3387(Medtronic), para estimulação cerebral profunda (ECP) com alta frequência, sendo este bilateral nos casos de TE e unilateral nos casos com tremor por DP. Resultados: Os pacientes tiveram seu seguimente clínico até o presente, com média de 12 meses, sendo observada a eficácia da estimulação do núcleo VIM no controle dos disparos das células do tremor. As complicações temporárias do tipo parestesias, disartrias e discreto aumento do tônus foram revertidas após o ajuste dos parâmetros de estimulação. Conclusão: Os resultados confirmam os achados da literatura, de que a estimulação talâmica é excelente opção terapêutica no tratamento do tremor, havendo possibilidade de estimulação talâmica bilateral simultânea com segurança.
\end{abstract}

PALAVRAS-CHAVE: estimulação cerebral profunda, núcleo VIM talâmico, tremor essencial, tremor parkinsoniano.

\section{Deep brain stimulation of VIM thalamic nucleus for tremor control}

ABSTRACT - Purpose: We present our results in 4 patients with tremor, in whom electrodes (uni and bilateral) for Deep Brain Stimulation (DBS) were implanted in the ventral intermediate nucleus (VIM) of the thalamus. Method: Four patients with disabling tremor, with drug-resistant spite of optimum therapeutic trials with poor response were referred to do surgery. Two patients had bilateral essential tremor. These patients were implanted with electrodes for DBS 3387 (Medtronic). Two patients had unilateral parkinsonian tremor and they received unilateral implantation of model 3387 DBS. Results: All four patients showed relieve of the tremor symptoms with significant tremor control seen at the scores. There were no definite adverse events after the electrodes implants for DBS; adverse events were transient and promptly reversed after the adjustment of the parameters. Conclusions: The results the authors found in this study indicate that VIM-stimulation is effective for tremor control either parkinsonian or essential tremor. The results correlate with the data in the literature.

KEY WORDS: deep brain stimulation, VIM, essential tremor, Parkinson disease.

A doença de Parkinson (DP) idiopática e o tremor essencial (TE) são doenças nas quais o tremor é um sinal ou sintoma fundamental, sendo muitas vezes incapacitante e em alguns casos refratários ao tratamento medicamentoso adequado. Desde a década de 50 que são conhecidos os efeitos das lesões terapêuticas talâmicas, especialmente do núcleo ventral interno (VIM), na diminuição dos diversos tipos de tremores $^{1,2}$. Todavia, são observadas diversas reações adversas, entre as mais comuns podemos citar as hemiparesias, disestesias, hipotonias e disartrias (em procedimentos bilaterais) e distúrbios cognitivos graves e permanentes ${ }^{3}$.

A estimulação cerebral profunda (ECP) talâmica vem se tornando uma alternativa no tratamento dos distúrbios do movimento. Na década de 80, Mazars et al. demonstraram a eficácia da ECP no controle das discinesias causadas pela deaferentação sensitiva $^{4,5}$. Atualmente, a ECP se tornou uma realidade, com seu emprego mais amplo nos distúrbios do mo-

\footnotetext{
'Serviço de Neurocirurgia do Hospital Pró-Cardíaco, Rio de Janeiro - Estereolife, Neurocirurgia Funcional e Estereotáxica, Rio de Janeiro - Instituto Neurológico Estácio de Sá (Instituto do Sono) Universidade Estácio de Sá , Rio de Janeiro RJ, Brasil; ${ }^{2}$ Doutorando pela Escola Paulista de Medicina, Universidade Federal de São Paulo, São Paulo SP, Brasil (EPM-UNIFESP); ${ }^{3}$ Pós-Graduação em Neurocirurgia EPMUNIFESP.
}

Recebido 21 Setembro 2001, recebido na forma final 12 Dezembro 2001. Aceito 10 Janeiro 2002.

Dr. José Augusto Nasser - Avenida Ataulfo de Paiva, 1079/1001 - 22440-031 Rio de Janeiro RJ - Brasil. FAX: 212945329. www.estacio.br/ines. E-mail: nasser@riodejaneiro.net 
vimento, permitindo o auxílio no controle dos demais sintomas parkinsonianos, estimulando o globo pálido interno ou o núcleo subtalâmico de Luys. Os resultados iniciais também mostram um controle semelhante à ablação, nos casos de distonia primária, DYT1 positivo, estimulando o globo pálido interno bilateralmente ${ }^{6,7}$. As técnicas de estimulação permitem o ajuste dos parâmetros elétricos a fim de obter controle adequado do tremor. Caso ocorram efeitos indesejáveis, estes podem ser prontamente revertidos através da mudança nos parâmetros de programação dos estímulos.

Apresentamos nessa experiência inicial com ECP do VIM para o controle do tremor e comparamos os resultados com as demais séries publicadas.

\section{MÉTODO}

\section{Avaliação clínica}

No período de outubro de 1999 a janeiro de 2001, foram estudados 4 pacientes portadores de tremor refratário a farmacoterapia e que não haviam sido tratados com cirurgia ablativa prévia.

Dois pacientes com DP apresentavam a forma "tremordominante" de alta amplitude, sendo um no dimídio direito e no esquerdo. As características dos 2 pacientes com DP eram respectivamente: - Paciente 1: 52 anos, sexo masculino, diagnóstico de DP há 5 anos, em tratamento com carbidopa/levodopa com dose total $01 \mathrm{~g} / \mathrm{dia}$. - Paciente 2: 58 anos, sexo masculino, diagnóstico de DP há 6 anos em tratamento com benzerazida/levodopa com dose total $01 \mathrm{~g} / \mathrm{dia}$.

Os outros dois pacientes selecionados para esse estudo apresentavam-se com diagnóstico de TE. Paciente 1: 62 anos, sexo feminino, em tratamento com betabloqueador $130 \mathrm{mg} /$ dia (regular) e primidona $125 \mathrm{mg} /$ dia. Paciente $2:$ 64 anos, sexo masculino, 20 anos de doença e com as mesmas dosagens dos medicamentos do paciente 1, mantinha seu tremor com alta amplitude comprometendo sua qualidade de vida.

Os pacientes com DP foram avaliados pelos autores no pré-operatório e no pós-operatório, sob efeito de medicamento (ON) e sem efeito de medicamento por 12 horas (OFF) aplicando-se a escala (UPDRS parte III) para avaliação do tremor ${ }^{8}$. Para os pacientes com TE foi aplicada, antes e após a cirurgia, a escala de pontuação clínica para o tremor (CRST) ${ }^{9}$.

\section{Procedimento Cirúrgico}

Planejamento estereotáxico feito com estereotomografia e em seguida as imagens transferidas para a central de trabalho, onde foram fundidas com às da ressonância magnética do cérebro do paciente feitas na véspera. Através do programa MSPS v5.08 (MEVIS - São Paulo SP - Brasil) foram feitas as fusões em tempo real. Em seguida as imagens eram superpostas ao atlas neurofuncional (Shaltem-
brand-Wharen) para através das coordenadas estereotáxicas específicas localizar com precisão o núcleo VIM talâmico.

O alvo estereotáxico aplicado para o VIM: plano ACPC ou $1 \mathrm{~mm}$ acima , 0-4 $\mathrm{mm}$ posterior ao ponto PMC e 12$13 \mathrm{~mm}$ lateral ao centro do III ventrículo. As coordenadas do alvo foram passadas para o sistema estereotáxico ETM03B (MICROMAR - São Paulo SP - Brasil). Com anestesia local foi feita uma trepanação frontal bilateral nos pacientes com TE e unilateral nos com DP, sendo posicionado um macroeletrodo (Radionics - MA - EUA) no VIM e posteriormente um microeletrodo verificando as respostas neurofisiológicas adequadas no dimídio contralateral ao procedimento e às vezes ipsilateral. Após a confirmação do melhor posicionamento da área a ser estimulada foi implantado um eletrodo quadripolar (modelo 3387, Minneapolis, MN - USA) e confirmado seu posicionamento final pela fluoroscopia em antero-posterior e perfil (Fig 1). A seguir foi feito a estimulação bipolar com programador Medtronic modelo 3625 com freqüências de $100-200 \mathrm{~Hz}$ e amplitude de pulso de 50-100 $\mu$ sec. A posição do microeletrodo foi assegurada quando a estimulação induziu discinesias, quando cessou o tremor, ou quando se desenvolveu contração tônica a 4.0V. No mesmo tempo os cabos foram conectados à extensão e ao gerador de pulso subclavicular (Itrel II). Em média oito dias após o procedimento é então realizada a programação inicial com programador externo (Medtronic - MN - EUA). Nos pacientes com TE foram implantados dois eletrodos independentes, com geradores independentes no núcleo VIM de cada lado. Nestes pacientes os tremores foram gradativamente sendo controlados.

Todos os pacientes foram submetidos à imagem por ressonância no pós-operatório confirmando o posicionamento do eletrodo, antes de fazer a programação (Fig 2).

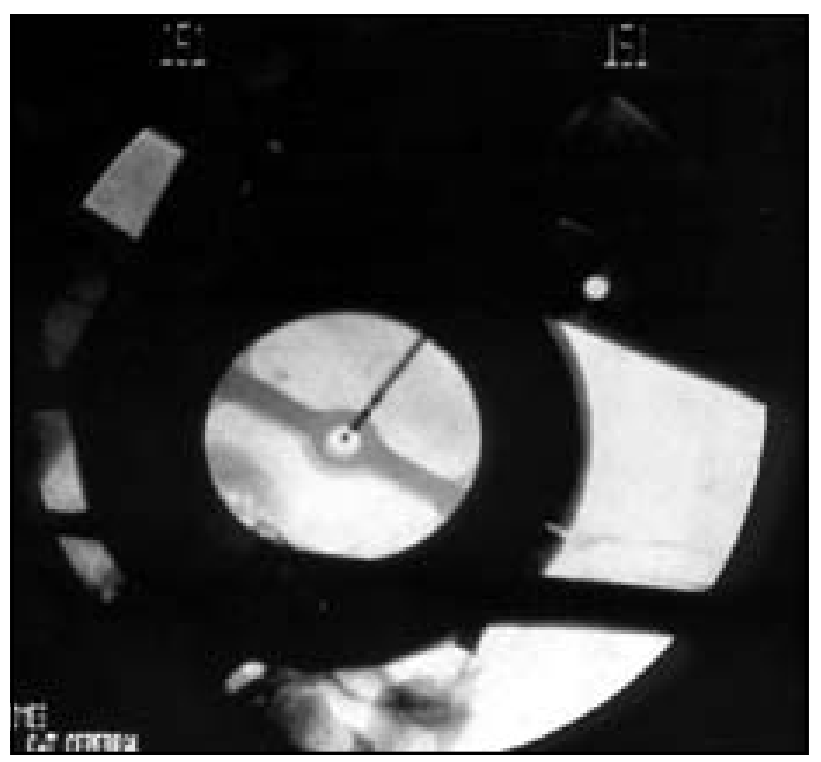

Fig 1. Fluoroscopia intraoperatória comprovando o correto posicionamento do eletrodo no alvo (núcleo VIM talâmico) após fixação do eletrodo quadripolar. 


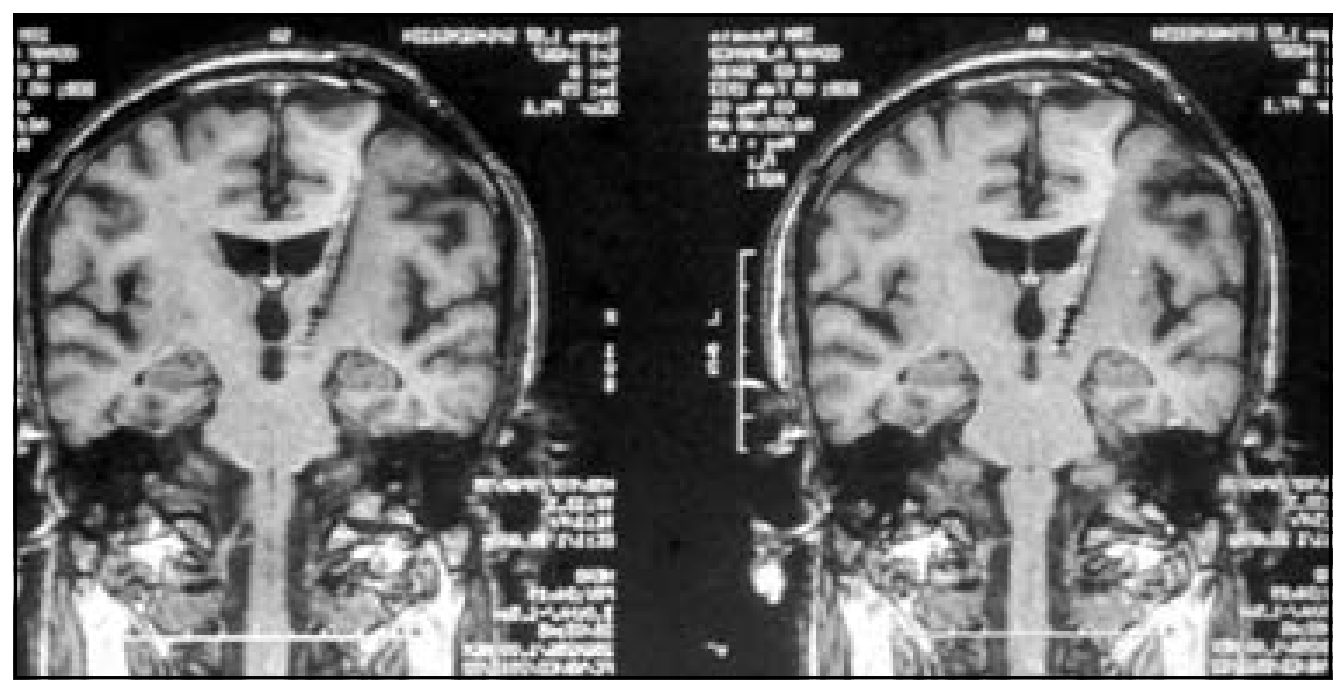

Fig 2. Ressonância magnética feita antes da primeira programação da estimulação cerebral comprovando o correto posicionamento do eletrodo no núcleo VIM talâmico esquerdo.

Tabela 1. Avaliação do primeiro paciente com DP tremor dominante, 3 meses e 1 ano após a cirurgia. UPDRS para tremor.

\begin{tabular}{|c|c|c|c|c|}
\hline Tremor & Variação & $\begin{array}{c}\text { Valor } \\
\text { pré- } \\
\text { operatório }\end{array}$ & $\begin{array}{c}3 \text { meses } \\
\text { pós- } \\
\text { operatório }\end{array}$ & $\begin{array}{c}\text { 1ano } \\
\text { pós- } \\
\text { operatório }\end{array}$ \\
\hline Mandíbula & $0-4$ & 2 & 0 & 0 \\
\hline $\begin{array}{l}\text { Tremor } \\
\text { de repouso } \\
\text { braço } \\
\text { contralateral }\end{array}$ & $0-4$ & 4 & 1 & 1 \\
\hline $\begin{array}{l}\text { Tremor } \\
\text { de repouso } \\
\text { perna } \\
\text { contralateral }\end{array}$ & $0-4$ & 4 & 0 & 0 \\
\hline $\begin{array}{l}\text { Tremor } \\
\text { de repouso } \\
\text { braço } \\
\text { ipsilateral }\end{array}$ & $0-4$ & 2 & 2 & 2 \\
\hline $\begin{array}{l}\text { Tremor } \\
\text { de repouso } \\
\text { perna } \\
\text { ipsilateral }\end{array}$ & $0-4$ & 1 & 1 & 2 \\
\hline $\begin{array}{l}\text { Tremor } \\
\text { de ação/ } \\
\text { postural } \\
\text { contralateral }\end{array}$ & $0-4$ & 0 & 0 & 0 \\
\hline $\begin{array}{l}\text { Tremor } \\
\text { de ação/ } \\
\text { postural } \\
\text { ipsilateral }\end{array}$ & $0-4$ & 0 & 0 & 0 \\
\hline
\end{tabular}

\section{RESULTADOS}

Quatro pacientes foram submetidos ao implante de eletrodo para ECP do núcleo VIM do tálamo.

A estimulação inicial proposta é sempre unipolar, sendo o objetivo usar o contacto que proporcione
Tabela 2. Avaliação do segundo paciente com DP tremor dominante, 3 meses e 1 ano após a cirurgia. UPDRS para tremor.

\begin{tabular}{|c|c|c|c|c|}
\hline Tremor & Variação & $\begin{array}{c}\text { Valor } \\
\text { pré- } \\
\text { operatório }\end{array}$ & $\begin{array}{c}3 \text { meses } \\
\text { pós- } \\
\text { operatório }\end{array}$ & $\begin{array}{c}1 \text { ano } \\
\text { pós- } \\
\text { operatório }\end{array}$ \\
\hline Mandíbula & $0-4$ & 0 & 0 & 0 \\
\hline $\begin{array}{l}\text { Tremor } \\
\text { de repouso } \\
\text { braço }\end{array}$ & & & & \\
\hline contralateral & $0-4$ & 4 & 0 & 0 \\
\hline $\begin{array}{l}\text { Tremor } \\
\text { de repouso } \\
\text { perna }\end{array}$ & & & & \\
\hline contralateral & $0-4$ & 4 & 1 & 0 \\
\hline $\begin{array}{l}\text { Tremor } \\
\text { de repouso } \\
\text { braço }\end{array}$ & & & & \\
\hline ipsilateral & $0-4$ & 1 & 1 & 1 \\
\hline $\begin{array}{l}\text { Tremor } \\
\text { de repouso } \\
\text { perna }\end{array}$ & & & & \\
\hline ipsilateral & $0-4$ & 0 & 0 & 0 \\
\hline $\begin{array}{l}\text { Tremor } \\
\text { de ação/ } \\
\text { postural } \\
\text { contralateral }\end{array}$ & $0-4$ & 0 & 0 & 0 \\
\hline $\begin{array}{l}\text { Tremor } \\
\text { de ação/ } \\
\text { postural } \\
\text { ipsilateral }\end{array}$ & $0-4$ & 0 & 0 & 0 \\
\hline
\end{tabular}

a melhor resposta ao tremor e menos efeitos secundários e com voltagem abaixo de 3V. Em todas as situações foi possível manter a estimulação permanente com uma amplitude abaixo de $3 \mathrm{~V}$ e com resposta clínica eficaz. 
Tabela 3. Avaliação pela CRST do primeiro paciente com tremor essencial bilateral, 3 meses e 1 ano após a cirurgia.

\begin{tabular}{|c|c|c|c|c|}
\hline Categoria & Variação & $\begin{array}{c}\text { Valor } \\
\text { pré-operatório }\end{array}$ & $\begin{array}{c}3 \text { meses } \\
\text { pós-operatório }\end{array}$ & $\begin{array}{c}1 \text { ano } \\
\text { pós-operatório }\end{array}$ \\
\hline CRST Total & $0-152$ & 68 & 22 & 19 \\
\hline Análise Subjetiva & $0-36$ & 22 & 9 & 8 \\
\hline Fala & $0-4$ & 2 & 1 & 1 \\
\hline Vestir & $0-4$ & 3 & 1 & 1 \\
\hline Beber & $0-4$ & 1 & 1 & 1 \\
\hline Ingestão sólida & $0-4$ & 3 & 1 & 1 \\
\hline Higiene & $0-4$ & 2 & 1 & 1 \\
\hline Escrever & $0-4$ & 4 & 1 & 1 \\
\hline Trabalhar & $0-4$ & 3 & 1 & 1 \\
\hline Dificuldade Global & $0-4$ & 4 & 2 & 1 \\
\hline Tremor & $0-84$ & 20 & 6 & 5 \\
\hline \multicolumn{5}{|l|}{ Repouso } \\
\hline Tremor repouso braço contralateral & $0-4$ & 1 & 0 & 0 \\
\hline Tremor repouso perna contralateral & $0-4$ & 0 & 0 & 0 \\
\hline Tremor repouso braço ipisilateral & $0-4$ & 1 & 1 & 1 \\
\hline Tremor repouso perna ipsilateral & $0-4$ & 0 & 0 & 0 \\
\hline \multicolumn{5}{|l|}{ Postural } \\
\hline Tremor postural braço contralateral & $0-4$ & 4 & 1 & 0 \\
\hline Tremor postural perna contralateral & $0-4$ & 1 & 0 & 0 \\
\hline Tremor postural braço ipsilateral & $0-4$ & 3 & 2 & 2 \\
\hline Tremor postural perna ipsilateral & $0-4$ & 0 & 0 & 0 \\
\hline \multicolumn{5}{|l|}{ Ação } \\
\hline Tremor ação braço contralateral & $0-4$ & 4 & 0 & 0 \\
\hline Tremor ação perna contralateral & $0-4$ & 2 & 0 & 0 \\
\hline Tremor ação braço ipsilateral & $0-4$ & 2 & 1 & 1 \\
\hline Tremor ação perna ipsilateral & $0-4$ & 0 & 0 & 0 \\
\hline Tremor Vocal & $0-4$ & 2 & 1 & 1 \\
\hline Escrever, desenhar, despejar & $0-32$ & 26 & 7 & 6 \\
\hline Escrever & $0-8$ & 7 & 2 & 2 \\
\hline Despejar & $0-8$ & 6 & 2 & 2 \\
\hline Desenho espiral & $0-8$ & 7 & 1 & 1 \\
\hline Desenho linear & $0-8$ & 6 & 2 & 1 \\
\hline
\end{tabular}

Os parâmetros médios de estimulação após um ano são: 1 contato unipolar: amplitude: $2.5 \mathrm{~V}$, largura de pulso: $120 \mu$ s e frequência: $185 \mathrm{~Hz}$. A impedância variou no início de $783 \Omega$ até $1.032 \Omega$ em 1 ano, o que faz com que, normalmente, haja uma necessidade de aumento na intensidade de corrente ao longo do tempo. Porém isto nem sempre foi observado.

Como mostram as Tabelas 1, 2, 3, 4 houve meIhora significativa nos 4 pacientes.

Nos casos de tremor por DP houve um controle acima de $85 \%$ após um ano de avaliação no lado oposto ao implantado e pouco efeito homolateral. Observa-se no paciente 1 um controle do tremor mandibular, efeito pouco comum quando a estimulação é apenas unilateral. As Tabelas 1 e 2 mostram apenas o UPDRS pré e pós-operatório relativos à avaliação do tremor. Contudo todos os pacientes com DP foram submetidos à avaliação completa do UPDRS e mostram que o ECP no VIM não altera de forma significativa nenhum outro sintoma da DP.
Nos casos de TE os resultados são similares nos 2 pacientes com discreta vantagem para o segundo paciente. Esta diferença não está ligada ao controle do tremor, mas na análise subjetiva e nas funções executivas.

Os efeitos adversos notificados foram do tipo parestesias, disartrias, discreto aumento do tônus contralateral e pressão nas órbitas que regrediram com a diminuição na amplitude (voltagem) do estimulador.

Não houve complicações operatórias maiores e permanentes. Estas não são infreqüentes na literatura, especialmente com maior número de trajetos dos microeletrodos.

\section{DISCUSSÃO}

Na década de 70, Hosobushi introduziu a estimulação cerebral profunda para dor intratável visando estimular os núcleos sensoriais do tálamo ${ }^{10}$. No início dos anos 80 Mazars considerava sua utilização em movimentos anormais ${ }^{5}$. Na metade da década 
Tabela 4. Avaliação pela CRST do segundo paciente com tremor essencial bilateral, 3 meses e 1 ano após a cirurgia.

\begin{tabular}{|c|c|c|c|c|}
\hline Categoria & Variação & $\begin{array}{c}\text { Valor } \\
\text { pré-operatório }\end{array}$ & $\begin{array}{c}3 \text { meses } \\
\text { pós-operatório }\end{array}$ & $\begin{array}{c}1 \text { ano } \\
\text { pós-operatório }\end{array}$ \\
\hline CRST Total & $0-152$ & 78 & 15 & 15 \\
\hline Análise Subjetiva & $0-36$ & 23 & 5 & 5 \\
\hline Fala & $0-4$ & 3 & 0 & 0 \\
\hline Vestir & $0-4$ & 3 & 1 & 1 \\
\hline Beber & $0-4$ & 3 & 1 & 1 \\
\hline Ingestão sólida & $0-4$ & 3 & 0 & 0 \\
\hline Higiene & $0-4$ & 3 & 0 & 0 \\
\hline Escrever & $0-4$ & 3 & 1 & 1 \\
\hline Trabalhar & $0-4$ & 4 & 1 & 1 \\
\hline Dificuldade Global & $0-4$ & 4 & 1 & 1 \\
\hline Tremor & $0-84$ & 24 & 5 & 5 \\
\hline \multicolumn{5}{|l|}{ Repouso } \\
\hline Tremor repouso braço contralateral & $0-4$ & 0 & 0 & 0 \\
\hline Tremor repouso perna contralateral & $0-4$ & 1 & 0 & 0 \\
\hline Tremor repouso braço ipisilateral & $0-4$ & 1 & 1 & 1 \\
\hline Tremor repouso perna ipsilateral & $0-4$ & 0 & 0 & 0 \\
\hline \multicolumn{5}{|l|}{ Postural } \\
\hline Tremor postural braço contralateral & $0-4$ & 4 & 0 & 0 \\
\hline Tremor postural perna contralateral & $0-4$ & 2 & 0 & 0 \\
\hline Tremor postural braço ipsilateral & $0-4$ & 4 & 2 & 2 \\
\hline Tremor postural perna ipsilateral & $0-4$ & 0 & 0 & 0 \\
\hline \multicolumn{5}{|l|}{ Ação } \\
\hline Tremor ação braço contralateral & $0-4$ & 4 & 0 & 0 \\
\hline Tremor ação perna contralateral & $0-4$ & 2 & 0 & 0 \\
\hline Tremor ação braço ipsilateral & $0-4$ & 3 & 1 & 1 \\
\hline Tremor ação perna ipsilateral & $0-4$ & 0 & 0 & 0 \\
\hline Tremor Vocal & $0-4$ & 3 & 1 & 1 \\
\hline Escrever, desenhar, despejar & $0-32$ & 31 & 5 & 5 \\
\hline Escrever & $0-8$ & 8 & 1 & 1 \\
\hline Despejar & $0-8$ & 7 & 2 & 2 \\
\hline Desenho espiral & $0-8$ & 8 & 1 & 1 \\
\hline Desenho linear & $0-8$ & 8 & 1 & 1 \\
\hline
\end{tabular}

de 80, na França, Benabid propôs o uso da estimulação por alta freqüência do núcleo VIM do tálamo no tratamento de tremores refratários ao tratamento farmacológico e onde a ablação poderia ser problemática, como é o caso da lesão bilateral do tálamo , do globo pálido e subtálamo ${ }^{11}$.

A literatura mostra que a estimulação somente do VIM não é ideal nos pacientes com DP sem a forma tremor-dominante ${ }^{6,7}$. O uso da ECP para o tratamento dos sintomas motores da DP requer um alvo mais completo, que também surta efeito nos outros sintomas cardinais da DP. Nesses casos, o globo pálido interno e o núcleo subtalâmico de Luys se prestam mais e têm seus resultados já bem estabelecidos na literatura ${ }^{12,13}$.

Benabid et al. avaliando 80 pacientes com DP e 20 com TE concluiram que ECP do VIM é altamente eficaz para controle de tremor, com $90 \%$ de redução contralateral em um seguimento de $1 \mathrm{ano}^{14}$. Koller et al. mostraram em seu estudo multicêntrico uma melhora acima de $80 \%$ no seguimento de 1 ano do tremor contralateral em 24 pacientes com DP e 29 com TE implantados com ECP do VIM ${ }^{15}$. O tratamento promoveu uma melhora considerável nas disfunções motoras nos casos de TE, porém este efeito não pode ser observado nos dois pacientes com DP e seus sintomas parkinsonianos não tremulantes.

Estes resultados, juntamente com os de Kumar, confirmam os efeitos do ECP nos pacientes com DP apenas para o tremor $^{16}$. Nota-se frequentemente uma discreta recrudescência do tremor em situações de estresse intenso, cessando após o episódio, algumas vezes é necessária a reprogramação. Isto foi observado nesta série e confirmado nos 2 pacientes apresentados. Quanto à análise dos pacientes com TE e ECP do VIM , houve uma maior melhora da fala e dos tremores cervicais nesta casuística comparada com a de Kumar ${ }^{16}$. Possivelmente se deva ao fato destes dois casos apresentarem TE bilateral e cirurgia bilateral, o que não ocorreu na totalidade dos casos 
de Kumar. Estes sintomas parecem apresentar meIhora mais expressiva após ECP bilateral. Mas o restante da análise subjetiva, em relação ao tremor de ação/postural e às atividades executivas, os resultados são excelentes e superponíveis.

Dois estudos recentes, um de Carpenter et al. e outro de Koller et al., têm mostrado efeitos positivos da ECP do VIM e TE, no tremor cervical e vocal, depois de cirurgia unilateral ${ }^{15,17}$. Taha et al. e Obwegeser et al. discordam desta afirmação e sugerem cirurgia bilateral para essas formas de tremor ${ }^{18,19}$. Nossos casos confirmam os achados de cirurgia bilateral para o controle dos tremores de linha média.

Atualmente é bem aceito que a estimulação do VIM promova efeitos similares aos da talamotomia, sem seus efeitos adversos e seus riscos de lesão permanente $\mathrm{e}^{20-23}$. Mas o mecanismo de ação da ECP ainda é objeto de pesquisa ${ }^{24-26}$. Bloqueio neuronal, modulação e inibição são as possibilidades. Estudos em tomografia por emissão de pósitrons indicam que a ECP do VIM causa desativação do cerebelo contralateral ao tremor. Todavia não é claro se isto é necessário para a supressão do tremor ou se esta supressão cerebelar acontece porque o tremor pára ${ }^{27}$.

Caparros-Lefebvre mostrou que a estimulação crônica do núcleo VIM promove poucas alterações patológicas próximas à área da lesão e a eficiência da estimulação mantem-se ao longo do tempo ${ }^{28}$.

\section{CONCLUSÕES}

A ECP do núcleo VIM talâmico é uma ferramenta terapêutica extremamente eficaz no tratamento dos tremores na doença de Parkinson e no tremor essencial.

A principal vantagem dos procedimentos neuroaumentativos, ECP, quando comparados aos métodos ablativos é a segurança, reversibilidade e adaptação. Estas vantagens fazem com que mesmo aparecendo efeitos adversos, estes podem ser contornados e prontamente revertidos.

A ECP do VIM do tálamo é um procedimento consagrado pela eficiência. Pode ser implantado bilateralmente com segurança e evitando as sequelas das talamotomias bilaterais.

\section{REFERÊNCIAS}

1. Talairach J, Paillas JE, David M. Dyskinésie de type hémiballique traitée par cortectomie frontale limitée, puis par coagulation de l'anse lenticulaire et de la portion interne du globus pallidus: amélioration importante depuis un an. Rev Neurol 1950; 83:440-451.

2. Narabayashi H. Neurophysiological ideas on pallidotomy and ventrolateral thalamotomy for hyperkinesias. Conf Neurol 1962;22:291-303.

3. Rossitch E Jr, Zeidman SM, Nashold BS Jr. Evaluation of memory and language function pre- and post-thalamotomy with an attempt to define those patients at risk for postoperative dysfunction. Surg Neurol 1998; 29:11-16.
4. Mazars G, Merienne L, Cioloca C. Treatment of certain types of pain with implantable thalamic stimulators. Neurochirurgie 1974;20:117-122.

5. Mazars G, Merienne L, Cioloca G. Control of dyskinesias due to sensory deafferentation by means of thalamic stimulation. Acta Neurochir (Wien) 1980;30(Suppl):239-243.

6. Pollak P, Benabid AL, Limousin P, Benazzouz A. The basal ganglia and new surgical approaches for Parkinson's disease. Adv Neurol 1997;74:213-220.

7. Kumar R, Lozano AM, Kim YJ, et al. Double-blind evaluation of subthalamic nucleus deep brain stimulation in advanced Parkinson's disease. Neurology 1998;51:850-855.

8. Fahn S, Elton R, Members of the UPDRS Development Committee. Unified Parkinson's disease rating scale. In Fahn S, Marsden C, Caine D, Goldstein M (eds). Recent developmentes in Parkinson's disease. London: MacMillan, 1987:153-164.

9. Fahn S, Tosola E, Martin C. Clinical rating scale for tremor. In Jankovic J, Tolosa E (eds). Parkinson's disease and movement disorders. Baltimore: Williams \& Wilkins, 1993:271-280.

10. Hosobuchi Y. Subcortical electrical stimulation for control of intractable pain in humans: report of 122 cases (1970-1984). J Neurosurg 1986;64:543-553.

11. Benabid AL, Pollak P, Louveau A, Henry S, De Rougemont J. Combined (thalamotomy and stimulation) stereotactic surgery of the VIM thalamic nucleus for bilateral Parkinson's disease. Appl Neurophysiol 1987;50:344-346.

12. Lang AE, Lozano AM. Parkinson's disease. N Engl J Med 1998;339:10441053.

13. Lang AE, Lozano AM. Parkinson's disease. N Engl J Med 1998;339:11301143.

14. Benabid AL, Pollak P, Gao DM, et al. Chronic electrical stimulation of the ventralis intermedius nucleus of the thalamus as a treatment of movement disorders. J Neurosurg 1996; 84:203-214.

15. Koller WC, Kelly EL, Wilkinson SB, Pahwa R. Efficacy of unilateral deep brain stimulation of the VIM nucleus of the thalamus for essential head tremor. Mov Disord 1999;14:847-850.

16. Kumar K, Kelly M, Toth C. Deep brain stimulation of the ventral intermediate nucleus of the thalamus for control of tremors in Parkinson's disease and essential tremor. Stereotact Funct Neurosurg 1999;72:4761.

17. Carpenter MA, Pahwa R, Miyawaki KL, Wilkinson SB, Seral JP, Koller WC. Reduction in voice tremor under thalamic stimulation. Neurology 1998;50:796-798.

18. Taha JM, Janszen MA, Favre J. Thalamic deep brain stimulation for the treatment of head, voice, and bilateral limb tremor. J Neurosurg 1999;91:68-72.

19. Obwegeser AA,Uitti RJ, Turk MF, Strongosky BA, Wharen RE. Thalamic stimulation for the treatment of midline tremors in essential tremor patients. Neurology 2000;54:2342-2344.

20. Jones MW, Tasker The relationship of documented destruction of specific cell types to complications and effectiveness in thalamatomy for tremor in Parkinson's disease. Stereotact Funct Neurosurg 1990;54:207211.

21. Jankovic J, Cardoso F, Grossman RG, Hamilton WJ. Outcome after stereotactic thalamotomy for parkinsonian, essential and others types of tremor. Neurosurgery 1995;37:680-87.

22. Lozano A. VIM thalamic stimulation for tremor. Arch Med Res 2000;31:266-269.

23. Nasser JA, Alaminos A, Ferraz, Confort CI. Preliminary results in surgery of Parkinson's disease. Arq Neuropsiquiatr 1998;56:533-539.

24. Paré D, Curro' Dossi R, Steriade M. Neuronal basis of the parkinsonian resting tremor: a hypothesis and its implications for treatment. Neuroscience 1990;35:217-226.

25. Albin R L, Young A B, Penney JB. The functional anatomy of basal ganglia disorders. TINS 1989;12:366-375.

26. Smith Y, Kieval JZ. Anatomy of the dopamine system in the basal ganglia. TINS 2000;23:28-33.

27. Pollak P, Benabid A, Limousin P, Benazzouz A. Chronic intracerebral stimulation in Parkinson's disease. Adv Neurol 1997;74:213-220.

28. Caparros-Lefebvre D, Ruchoux MM, Blond S, Petit H, Percheron G. Long-term thalamic stimulation in Parkinson's disease: postmortem anatomical study. Neurology 1994;44:1856.

29. Lucas J, Rippeth JD, Uitti RJ, Shuster EA, Wharen R. Neuropsychological functional in a patient with essential tremor with and without bilateral VIM stimulation. Brain and Cognition 2000;42:253-267. 